\title{
Facilitated Acquisition but Not Persistence of Responding for a Cocaine-Paired Conditioned Reinforcer Following Sensitization with Cocaine
}

\author{
Patricia Di Ciano*, \\ 'Department of Experimental Psychology, University of Cambridge, Cambridge, UK
}

Sensitization has been hypothesized to increase the incentive salience of drug-paired conditioned stimuli and in the present study the ability of a sensitizing pretreatment with cocaine to increase responding for a drug-paired conditioned reinforcer was tested. In support of the incentive-sensitization hypothesis, sensitized rats earned more presentations of a drug-paired conditioned reinforcer during acquisition of a new response for this stimulus. By comparison, sensitization had no effect on the number of CSs earned during reversal of the contingency or following pretreatment with $d$-amphetamine. During reversal learning, however, sensitized rats were impaired in the extinction of the inappropriate response once the contingent CS was no longer available following presses on that lever. The results are discussed with reference to a possible role of increased incentive salience of a drug-paired CS to the formation of a habit. Neuropsychopharmacology (2008) 33, |426- |431; doi: | 0.1038/sj.npp. I 30 I542; published online 22 August 2007

Keywords: cocaine; habit; sensitization; conditioning; self-administration; rat

\section{INTRODUCTION}

Drug 'craving' and urges can be induced by a variety of factors (Koob and Le Moal, 2001; Kalivas et al, 2005), and conditioned stimuli (CSs) previously paired with drugs of abuse are especially potent as such after prolonged abstinence from the drug (Gawin and Kleber, 1986). Following repeated administration of psychostimulants to animals, measures of motivated behavior are sensitized (Harmer and Phillips, 1998; Taylor and Horger, 1999; Wyvell and Berridge, 2000, 2001; Vezina et al, 2002), an effect that is believed to underlie the persistent and compulsive nature of drug urges induced by CSs (Robinson and Berridge, 1993). Indeed, sensitization increased the transfer of control over responding by a Pavlovian CS (Wyvell and Berridge, 2000, 2001).

The ability of CSs to support new learning is a critical test of their acquired motivational properties (Mackintosh, 1974 ) and a novel response supported by reward-paired conditioned reinforcers can be persistent (Zimmerman, 1957; Robbins, 1976; Shahan, 2002; Di Ciano and Everitt, 2004). Indeed, sensitization increased the potentiative effects of intra-nucleus accumbens infusions of $d$-amphetamine on the acquisition of a new response for a food-paired

* Correspondence. Current address: Dr P Di Ciano, Department of Psychology, School of Human Sciences, University of Wales, Singleton Park, Swansea, SA2 8PP, UK, Tel: + 4401792295908 , Fax: + 4401792 295679, E-mail: p.diciano@swansea.ac.uk

Received 27 June 2007; revised I4 July 2007; accepted 25 July 2007 conditioned reinforcer (Taylor and Horger, 1999). However, well-established (Adams and Dickinson, 1981) instrumental responding or responding for a conditioned reinforcer after systemic psychostimulants (Robbins, 1976) can become autonomous of the outcome, and this may be partly because the value of the CS may become independent of the drug with which it was originally paired (Di Ciano and Everitt, 2004). As such, established responding for a drug-paired conditioned reinforcer would not depend on the associated incentive salience of the CS and therefore would not be affected by sensitization.

The purpose of the present study was to test whether repeated administration of cocaine that has been previously shown to produce robust sensitization of locomotor activity (Vanderschuren and Kalivas, 2000; Vanderschuren et al, 2003), could facilitate the acquisition or persistence of responding for a drug-paired conditioned reinforcer. To that aim, rats were sensitized before the acquisition of a novel response for a conditioned reinforcer previously paired with cocaine, to test for an effect of sensitization on: (1) the acquisition of a lever press response for a drugpaired conditioned reinforcer, when responding is maintained by a stimulus-reward association (Di Ciano and Everitt, 2004); (2) subsequent potentiation of this response with systemic $d$-amphetamine, which can be associated with responding that is independent of the outcome (Robbins, 1976) or habitual; or (3) reversal of the contingency for the drug-paired conditioned reinforcer after the response was well established, as sensitization has been shown to affect reversal of a stimulus-reward association (Jentsch et al, 2002; Schoenbaum et al, 2004). 


\section{METHODS}

\section{Animals}

Twenty-four (12 per group) male Lister Hooded rats weighing $280-300 \mathrm{~g}$ at the time of surgery (Charles River; Ramsgate, Kent, UK) were individually housed under a reversed $12 \mathrm{~h}$ light-dark cycle (lights on at $2000 \mathrm{~h}$ ). Rats were maintained on a restricted diet of $20 \mathrm{~g}$ of Purina lab chow/day, sufficient to maintain body weight and growth throughout the experiment. Water was available freely and food was given within $2 \mathrm{~h}$ after daily testing. Experiments were carried out between 0900 and $2000 \mathrm{~h}, 6$ or 7 days a week. Experiments were conducted in accordance with the United Kingdom 1986 Animals (scientific procedures) Act.

\section{Apparatus}

Rats were tested in operant chambers $(29.5 \times 32.5 \times$ $23.5 \mathrm{~cm}$; Med Associates; Hampton, UK). Three sides were constructed from Perspex and the fourth was made of stainless steel, on which two 4 -cm-wide retractable levers were secured. The two levers were $12 \mathrm{~cm}$ apart, and $8 \mathrm{~cm}$ from the grid floor. Above each lever was a cue light $(2.5 \mathrm{~W}$, $24 \mathrm{~V})$, and a red house light $(2.5 \mathrm{~W}, 24 \mathrm{~V})$ was located on the opposite wall. A food entry port was located in the middle of the two levers, and each head entry (nose poke) was detected by interruption of an infrared beam. The floor of the chamber was lined with absorbent paper and covered with a metal grid. The testing chamber was placed within a sound- and light-attenuating box, equipped with a ventilation fan that also screened external noise. Silastic tubing (Altec; Cornwall, UK) shielded with a metal spring extended from each animal's i.v. catheter to a liquid swivel (Stoelting, Wood Dale, IL, USA) mounted on an arm fixed outside of the operant chamber. Tygon tubing (Altec; Cornwall, UK) extended from the swivel to a Razel infusion pump (Semat Technical Ltd., Herts, UK) located adjacent to the external chamber. The operant chamber was interfaced to software running on written by $\mathrm{RN}$ Cardinal in $\mathrm{C}++$ using the Whisker control system (Cardinal and Aitken, 2001).

\section{Catheter Surgery}

Rats were anaesthetized with ketamine hydrochloride (100 mg/kg intraperitoneal (i.p.); Ketaset; Dunlops, Dumfries, UK) and xylazine ( $9 \mathrm{mg} / \mathrm{kg}$ i.p.; Dunlops, Dumfires, $\mathrm{UK}$ ) and supplemented with ketamine as needed ( $\sim 20 \mathrm{mg}$ ). All rats were implanted with a single catheter in the right jugular vein aimed at the left vena cava. Catheters were purchased readymade and consisted of a $22 \mathrm{~g}$ cannulae attached to Silastic tubing (0.012 inner diameter) and fixed to nylon mesh (Camcaths; www.camcaths.com; Cambridge, UK). The mesh end of the catheter was sutured subcutaneously (s.c.) on the dorsum. All surgical instruments were thoroughly sterilized before surgery. To prevent infection, rats were treated post-surgically with $10 \mathrm{mg} / \mathrm{kg}$ Baytril (Dunlops, Dumfries, UK) s.c. for eight days.

\section{Training Procedure (see Figure 1)}

1. Rats were trained to associate cocaine infusions with the presentation of a CS during seven daily experimental tests that began 7-10 days after surgical procedures. On each testing day, rats were connected to the i.v. line before the start of the training session. During 7 days of training, rats received a single i.v. cocaine infusion $(0.25 \mathrm{mg} /$ infusion $/ 0.1 \mathrm{ml} / 5 \mathrm{~s})$ following every nosepoke into the magazine (fixed ratio 1 (FR-1), time-out $20 \mathrm{~s}$ ). Each cocaine infusion was accompanied with illumination of a stimulus light above one of the retracted levers for $20 \mathrm{~s}$. During this $20 \mathrm{~s}$ 'time out', the houselight was extinguished and nosepokes were recorded but had no consequences. Following this $20 \mathrm{~s}$ stimulus, the house light was again illuminated and the stimulus light was extinguished. The side of the stimulus light was counterbalanced between left and right sides for individual animals. 'Priming' injections of cocaine were never given. To prevent accidental overdose, rats were limited to 30 infusions in a $60 \mathrm{~min}$ session. Rats were never given experimenter-administered 'priming' injections of cocaine and were not pretrained to respond (either nose poke or lever press) for food.

2. After initial training to self-administer cocaine, rats were randomly assigned to either the sensitization or control group ( $n=12$ each) and the sensitization group was administered repeated cocaine in a dose and pattern that has previously demonstrated to produce robust psychomotor sensitization (Vanderschuren and Kalivas, 2000; Vanderschuren et al, 2003). The sensitization group received a daily injection with cocaine $(30 \mathrm{mg} / \mathrm{kg}$, i.p.) on 5 consecutive days. The control group received five daily injections with saline $(1 \mathrm{ml} / \mathrm{kg}$, i.p.). All injections were given in the home cage. Subsequently, the animals were left undisturbed in their home cages for 3 weeks.

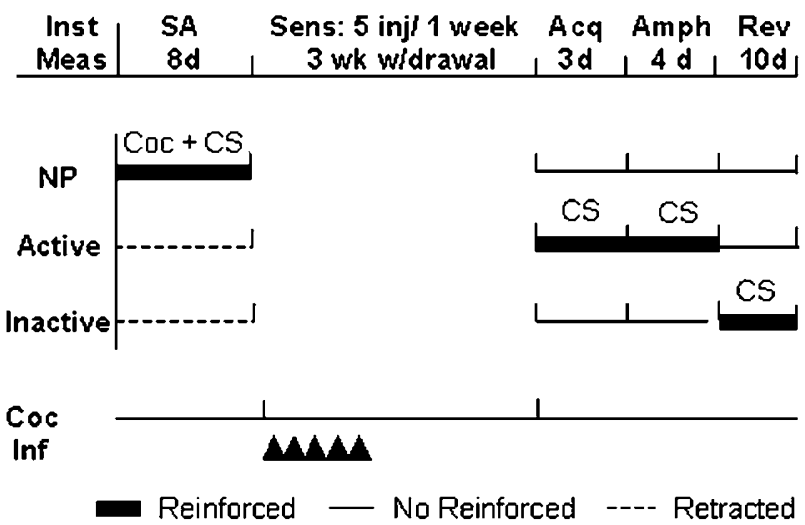

Figure I Schematic of sequence of behavioral testing. The instrumental measures (inst meas) are presented as reinforced (dark lines with the reinforcer specified above), recorded with no programmed consequences (thin lines) or retracted (stippled lines). Rats were trained to self-administer (SA) cocaine for 8 days (d) during which nose pokes (NP) resulted in cocaine (COC) infusions and associated conditioned stimulus (CS). Subsequently, rats were given sensitizing (sens) pretreatments of cocaine injections (inj) (vertical arrows) and left in their home cages for a further 3 weeks (wk) of withdrawal (w/drawal). At the test for acquisition of a lever press for a drug-paired conditioned reinforcer (Acq), two novel levers were inserted into the operant chamber for the first time and presses on the active lever resulted in presentations of the drug-paired conditioned reinforcer only. Rats were then challenged with systemic d-amphetamine (Amph) and the assignment of levers as active or inactive was reversed (Rev). 


\section{Tests for Sensitization (see Figure 1)}

1. Acquisition of a novel response for a drug-paired conditioned reinforcer: rats were placed in the operant chambers for four $30 \mathrm{~min}$ sessions and two novel levers were inserted; presses on the lever below the CS were reinforced with a $1 \mathrm{~s}$ presentation of the stimulus light previously paired with cocaine. Responses were reinforced under a variable ratio $1: 3$ schedule of reinforcement. During acquisition, nose pokes were measured but had no programmed consequences.

2. Potentiation of lever presses for a drug-paired conditioned reinforcer: rats were subsequently treated with four counterbalanced doses (Keppel, 1991) of $d$-amphetamine (veh, $0.25,0.50,1.0 \mathrm{mg} / \mathrm{kg}$ ) on consecutive days before responding for the conditioned reinforcer. $d$-Amphetamine was dissolved in sterile physiological saline and injected intraperitoneally, $15 \mathrm{~min}$ before testing, at a concentration of $1 \mathrm{mg} / \mathrm{ml}$.

3. Reversal learning with conditioned reinforcement: The day after $d$-amphetamine treatments the assignment of levers as active or inactive was reversed. The location of the conditioned reinforcer was kept constant.

\section{Statistical Analyses}

For all sessions, the number of CS presentations, lever presses or nose pokes was recorded. Data were analyzed with repeated-measures Group (two levels) $\times$ Day (seven levels for acquisition of the nose poke; three levels for acquisition of the lever press response and reversal of the contingency) or Group (two levels) $\times$ Dose (four levels) $\times$ Lever (two levels; active or inactive; potentiation by $d$ amphetamine) ANOVAs followed by planned comparisons on the effect of Group for each day; a criterion of $p<0.05$ was selected for significance.

\section{RESULTS}

Both groups of rats ( $n=12$ each in the control and tobe-sensitized groups) acquired the nose poke response for cocaine on the first day of self-administration and responding remained stable across days of self-administration training (Figure 2). Only an effect of measure (infusions or nose pokes) was found $(F(1,22)=37.19$, $p<0.001)$, suggesting that the number of nose pokes made was higher than the number of cocaine infusions earned.

During acquisition (Figure 3; top panels) of a novel lever press response for the drug-paired conditioned reinforcer and reversal of the contingency (Figure 3; bottom panels), rats readily acquired the lever press response (Figure $3 \mathrm{a}$ ) for the drug-paired conditioned reinforcer (CS; Figure 3d). During acquisition, responding by both groups on the active lever was higher than on the unreinforced inactive lever (Figure 3b; Lever: $\mathrm{F}(1,22)=4.353, p<0.001$ ), while the number of CS presentations was higher in the sensitized group $(n=12)$ than the vehicle group $(n=12$; Group: $\mathrm{F}(1,22)=5.377, p=0.030)$, which planned comparisons revealed were due to a Group difference on day 2 $(p<0.05)$. During acquisition of the new response for the CS, unreinforced nose pokes decreased in both groups of

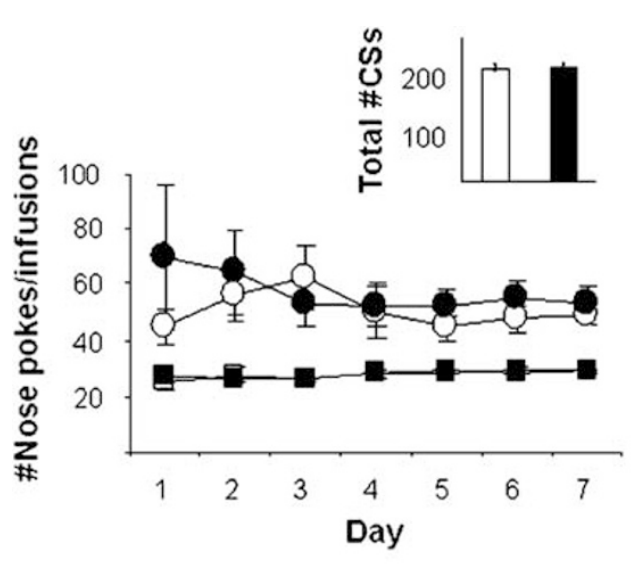

Responses: To-be-sensitized/control:

Figure 2 Mean \pm SEM number of nose pokes (circles) or cocaine infusions and associated light stimulus (squares) during the acquisition of cocaine self-administration during which the cocaine + CS association was formed. The to-be-sensitized rats (dark symbols; $n=12$ ) were not different from control rats (open symbols; $n=12$ ). Inset: Mean \pm SEM total number of cocaine infusions + CS pairings received during training.

rats (Figure 3c), as revealed by a significant one-way ANOVA on the effect of day $\mathrm{F}(2,44)=17.214, p<0.001)$. Planned comparisons revealed no significant effects.

During reversal of the contingency (Figure 3; bottom panels), the pattern of responding on the two levers varied differently across days for both groups of rats (lever $\times$ day: $\mathrm{F}(2,44)=15.492, \quad p<0.001)$, and this was due to a significant decrease in responding on the now-inactive lever (Figure 3b) after omission of the contingent CS (Figure 3d; day: $\mathrm{F}(2,44)=20.048, p<0.001)$, which planned comparisons revealed were due to a Group difference on day 2 only $(p<0.05)$. No significant effects were revealed for the active lever producing the CS (Figure 3a) or unreinforced nose pokes (Figure 3c). During reversal of the contingency, the number of CS presentations (Figure $3 \mathrm{~d}$ ) remained stable over days, and a day (three levels; days 1, 2, and 3) $\times$ Group (two levels; sensitized and control) ANOVA, with Group as the between-subjects factor, revealed no significant effects: (day: $\mathrm{F}(2,44)=1.281, p=0.288$; Group: $\mathrm{F}(1,22)=0.816, p=0.376$; interaction: $\mathrm{F}(2,44)=0.439$, $p=0.647)$.

Following administration of $d$-amphetamine (Figure 4), presses on both the active and inactive levers increased (Figure 4; left panel), and this increase was higher on the active lever, as revealed by a significant Dose $\times$ Lever interaction $(\mathrm{F}(3,66)=16.847, p<0.001)$. No significant effects were revealed for analysis of CS presentations.

\section{DISCUSSION}

The purpose of the present study was to evaluate the effects of sensitizing treatment with systemic cocaine on a lever press response for a drug-paired conditioned reinforcer. It was found that rats that received sensitizing pretreatment with drug received more earned presentations of the CS during acquisition of the novel response, with no effect of sensitization on the number of CS presentations earned after potentiation of responding by systemic 

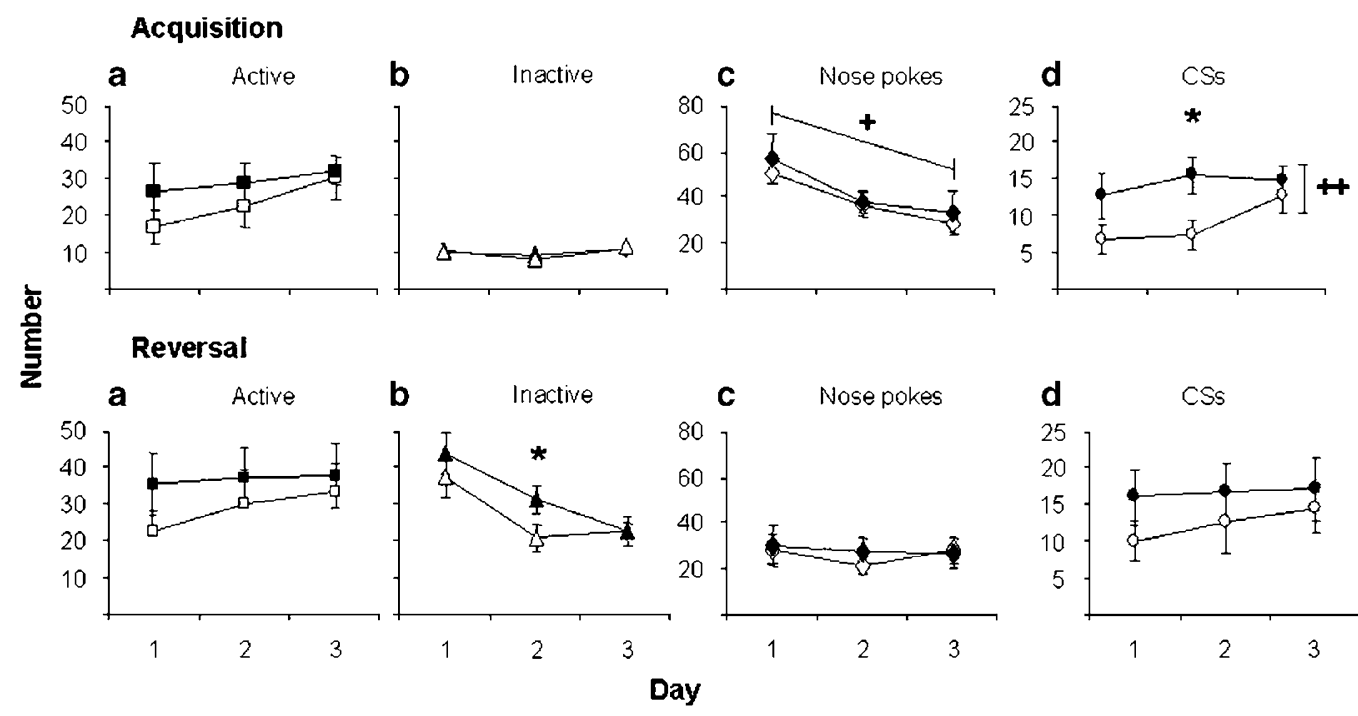

Control: $\square / \triangle / \otimes / O$

Sensitized:

Figure 3 Mean \pm SEM number of active lever presses producing the CS (a), unreinforced inactive lever presses (b), unreinforced nose pokes (c) or CS presentations (d) during the first 3 days of acquisition of a new response for a drug-paired conditioned reinforcer (top panels) or subsequent reversal of the contingency (bottom panels). Sensitized rats (dark symbols; $n=12$ ) received more CS presentations during acquisition than control rats (open symbols; $n=12$ ), and were slower to decrease responding on the now-inactive lever when the drug-paired conditioned reinforcer was no longer available following presses on that lever.,+++ and *indicate significant main effects of day, main effects of Group, and planned comparisons between Groups on that day, respectively $(p<0.05)$.

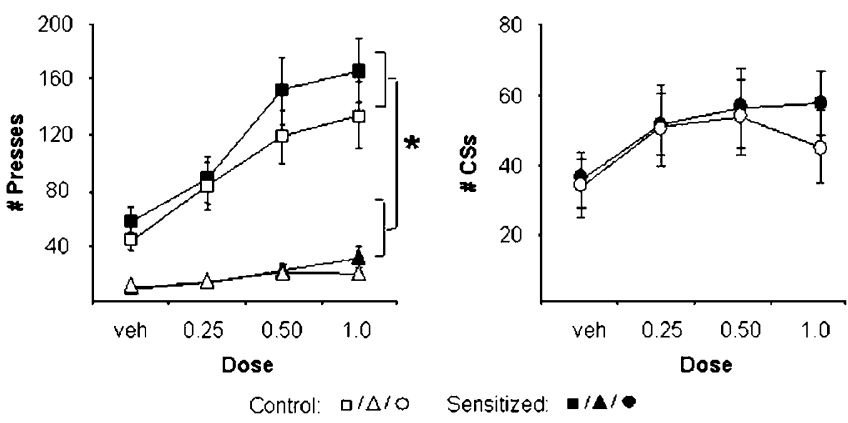

Figure 4 Mean \pm SEM number of lever presses (left panel; active lever = squares; inactive lever = triangles) or CS presentations (right panel; circles) following systemic pretreatment with d-amphetamine. No differences were revealed between sensitized (dark symbols; $n=12$ ) and control rats (open symbols; $n=12$ ). ${ }^{*}$ Indicates a significant day $\times$ lever interaction $(p<0.05)$.

$d$-amphetamine or during reversal of the contingency. However, the sensitized rats made more responses on the previously active lever when the contingency was reversed, despite showing no impairment on the acquisition of the new contingency for the drug-paired reinforcer. Sensitized rats were not impaired during the extinction of the nose poke response that originally produced cocaine and the associated stimulus during acquisition of this association, suggesting that the effects during reversal were related to a resistance to extinction of the response that was previously supported by the drug-paired conditioned reinforcer.

The acquisition of a new response by a reward-paired conditioned reinforcer is a stringent test of the acquired motivational properties of a CS (Mackintosh, 1974). In the present paradigm, the response made for the CS (lever press) is different from that made for the drug (nose poke), allowing for conditioned reinforcement to be measured in its own right, as there is no association between the response for the CS and the response for cocaine. This therefore provides an ideal test of the facilitatory influence of sensitization on the incentive motivational properties of drug-paired CSs per se (Robinson and Berridge, 1993). In the present study, both groups of rats readily acquired this response, consistent with previous findings (Di Ciano and Everitt, 2004). It is thus notable in the present study that during acquisition, sensitized rats received more earned presentations of the CS than controls, suggesting that sensitization increased the incentive properties of the CS, consistent with findings that sensitization increased transfer of control by a Pavlovian CS (Wyvell and Berridge, 2000, 2001).

By comparison, sensitization had no effect on the potentiation of responding for the drug-paired conditioned reinforcer following systemic administration of $d$-amphetamine. This is not consistent with the previous finding that sensitization facilitated the acquisition of a novel response for a food-paired CS following infusion of $d$-amphetamine into the NAcc (Taylor and Horger, 1999). However, it should be noted that in the present study the test for potentiation was conducted after the response had been established, and thus the findings by Taylor and Horger (1999) may reflect an impact of sensitization on the acquisition of this response. This underscores the suggestion that sensitization increases the incentive salience of CSs (Berridge and Robinson, 1998), which has been shown to be more important in the early stages of acquisition of an instrumental response (Adams, 1980, 1982; Adams and Dickinson, 1981).

Following reversal of the lever assignment as active or inactive, rats learned to respond appropriately for the drugpaired conditioned reinforcer on the previously inactive lever. The ability of sensitized rats to follow the new location of the conditioned reinforcer and thus acquire the appropriate new response suggests that the conditioned 
reinforcer was still maintaining responding. Yet, by comparison to initial acquisition of this response, sensitization had no effect on the number of CSs earned during reversal. Indeed, persistent responding for the conditioned reinforcer may develop a habitual (Robbins, 1976), 'autonomous' (Zimmerman, 1957) or 'automatic' (Tiffany, 1990) property over time, such that responding is no longer maintained by an associated value of the CS with the (drug) outcome. For example, extinction of the CS-cocaine association decreased the acquisition of responding for this CS but had little effect on established responding for the conditioned reinforcer (Di Ciano and Everitt, 2004). Sensitization has been hypothesized to increase the incentive salience of CSs (Berridge and Robinson, 1998), and thus in the present study sensitization did not affect responding supported by a conditioned reinforcer that may no longer be dependent on an association with the primary reward.

Sensitized rats were impaired in the extinction of the now-inappropriate response once the conditioned reinforcer was no longer available following that response, consistent with findings that sensitization impaired the reversal of the stimulus-reward associations (Jentsch et al, 2002; Schoenbaum et al, 2004), and facilitated a transition from response-outcome to habitual responding (Nelson and Killcross, 2006). However, in the present study, sensitized rats were not impaired in the extinction of the original nose poke response for cocaine once the cocaine and associated CS were no longer available following this response. Thus, the present findings cannot be interpreted simply in terms of extinction of the stimulus-reward association or impairments in preservative responding.

Instead, this may reflect some aspect of conditioned reinforcement that is resistant to extinction. It has been suggested that instrumental responding can develop an experience-dependent autonomy from the outcome that is context dependent (Dickinson, 1985). Responding for conditioned reinforcers is greater in contexts where animals have received reward (Shahan and Burke, 2004), and thus the conditioned reinforcer may form associations with the context during establishment of the response. Indeed, sensitization is context-dependent (Badiani et al, 1997) and in the present study the sensitized group may have formed relatively stronger associations between the context and the conditioned reinforcer during acquisition, due to increased incentive salience of the CS following sensitization.

It must be considered that the present results were confounded by cocaine self-administration experience (Phillips and Di Ciano, 1996; Mendrek et al, 1998; Morgan et al, 2006), as sensitization is context-dependent (Badiani et al, 1997) and both groups of rats self-administered cocaine in the testing chamber. This is not likely, however, as the sensitized rats received more CS presentations during acquisition of the novel response than the control group, and were slower to extinguish that response when the CS was no longer available. Consistent with this, sensitizing pretreatments of cocaine in the same dose and pattern used in the present study produced robust measures of sensitized psychomotor activation (Vanderschuren and Kalivas, 2000; Vanderschuren et al, 2003). Importantly, in the present study, the sensitized rats did not make more responses on the active lever, suggesting that sensitization increased the incentive properties of the CS and did not simply increase non-specific motor activity directed at the lever, arguing against the notion that lever pressing is simply an expression of locomotor activity.

Indeed, extended cocaine self-administration also sensitized a nose poke response for a cocaine-paired CS, and it was suggested that this was not related simply to a quantitative increase in behavior following sensitization (Ferrario et al, 2005). Indeed, in the present study, sensitizing pretreatment affected the number of CS presentations earned, despite much less cocaine selfadministration experience. The greater predisposition to sensitization in the present study may reflect differences in the incentive salience of outcomes in these two studies. That is, in the study by Ferrario et al (2005), the sensitized response for the CS had previously produced drug, and the animals were therefore working for drug (the outcome). In the present study, responding for the CS was not associated with a response for drug, and therefore provides a stringent test of the acquired motivational properties of the CS (Mackintosh, 1974) in its own right. Taken together, these studies suggest that sensitization of the incentive salience of a CS occurs following less cocaine experience than sensitized responding for drug, implicating the CS as vital to the acquisition of a drug habit, consistent with the incentive-sensitization theory (Robinson and Berridge, 1993, 2000; Berridge and Robinson, 1998).

\section{CONCLUSIONS}

The present findings provide direct support for the hypothesis that sensitization increases the incentive motivational properties of drug-paired CSs (Berridge and Robinson, 1998). In the present paradigm, responding for the drug-paired conditioned reinforcer is relatively more dependent on the stimulus-reward association, or incentive properties of the CS, when the animals are learning the novel response for the CS, as compared to responding that is well established, or persistent (Di Ciano and Everitt, 2004). The finding of the present study that sensitization enhanced the incentive properties of a drug-paired conditioned reinforcer during acquisition, with no effect on responding for the CS when the response was established, suggests that it is the incentive properties of the drug-paired CS that are increased by sensitization. The transition from actions to habits has been suggested to be a critical feature of addiction (Robbins and Everitt, 1999) and persistent responding for drug-paired conditioned reinforcers may develop habitual (Lyon and Robbins, 1975; Robbins, 1976), 'autonomous' (Zimmerman, 1957) or 'automatic' (Tiffany, 1990) properties over time. The present findings suggest that sensitization may form a critical role in the transition from drug use to drug abuse by enhancing the incentive properties of drug-paired CSs that can induce drug-related thoughts and behaviors.

\section{ACKNOWLEDGEMENTS}

This research was supported by an MRC Programme Grant no. G9536855 and was conducted within the University of Cambridge Behavioral and Clinical Neuroscience Institute supported by a joint award from the Medical Research 
Council and the Wellcome Trust. PDC would like to thank Professor Kent Berridge, Professor Trevor Robbins and Louk JMJ Vanderschuren for constructive comments on this manuscript and BJ Everitt who supported this research in his lab.

\section{DISCLOSURE/CONFLICT OF INTEREST}

The authors declare that this work was funded by MRC Programme Grant no. G9536855 to BJ Everitt, and anticipate receiving funding from NIDA R01 DA 20527-01A1 to PDC to support this program of research.

Not related to the present submission: The authors declare that over the past three years PDC has received honoraria from Pfizer and MasiMax Resources.

$P D C$

NIDA

Pfizer

MasiMax Resources

\section{REFERENCES}

Adams CD (1980). Post-conditioning devaluation of an instrumental reinforcer has no effect on extinction performance. Q J Exp Psychol 32: 447-458.

Adams CD (1982). Variations in the sensitivity of instrumental responding to reinforcer devaluation. Q J Exp Psychol 34B: 77-98.

Adams CD, Dickinson A (1981). Instrumental responding following reinforcer devaluation. Q J Exp Psychol 33B: 109-121.

Badiani A, Camp DM, Robinson TE (1997). Enduring enhancement of amphetamine sensitization by drug-associated environmental stimuli. J Pharmacol Exp Ther 282: 787-794.

Berridge KC, Robinson TE (1998). What is the role of dopamine in reward: hedonic impact, reward learning, or incentive salience? Brain Res Brain Res Rev 28: 309-369.

Cardinal RN, Aitken MRF (2001). Whisker (version 2.0). Cambridge University Technical Services: Cambridge.

Di Ciano P, Everitt BJ (2004). Conditioned reinforcing properties of stimuli paired with self-administered cocaine, heroin or sucrose: implications for the persistence of addictive behaviour. Neuropharmacology 47(Suppl 1): 202-213.

Dickinson A (1985). Actions and habits: the development of behavioural autonomy. Philos Trans $R$ Soc Lond 308: 67-78.

Ferrario CR, Gorny G, Crombag HS, Li Y, Kolb B, Robinson TE (2005). Neural and behavioral plasticity associated with the transition from controlled to escalated cocaine use. Biol Psychiatry 58: 751-759.

Gawin FH, Kleber HD (1986). Abstinence symptomatology and psychiatric diagnosis in cocaine abusers. Clinical observations. Arch Gen Psychiatry 43: 107-113.

Harmer CJ, Phillips GD (1998). Enhanced appetitive conditioning following repeated pretreatment with d-amphetamine. Behav Pharmacol 9: 299-308.

Jentsch JD, Olausson P, De La Garza II R, Taylor JR (2002). Impairments of reversal learning and response perseveration after repeated, intermittent cocaine administrations to monkeys. Neuropsychopharmacology 26: 183-190.

Kalivas PW, Volkow N, Seamans J (2005). Unmanageable motivation in addiction: a pathology in prefrontal-accumbens glutamate transmission. Neuron 45: 647-650.

Keppel G (1991). Design and Analysis. A Researcher's Handbook. Prentice Hall: Englewood Cliffs, NJ.

Koob GF, Le Moal M (2001). Drug addiction, dysregulation of reward, and allostasis. Neuropsychopharmacology 24: 97-129.
Lyon M, Robbins TW (1975). The action of central nervous system stimulant drugs: a general theory concerning amphetamine effects. In: Essman WB, Valzelli L (eds). Current Developments in Psychopharmacology. Spectrum Publications: New York. pp 79-163.

Mackintosh NJ (1974). The Psychology of Animal Learning. Academic Press: London.

Mendrek A, Blaha CD, Phillips AG (1998). Pre-exposure of rats to amphetamine sensitizes self-administration of this drug under a progressive ratio schedule. Psychopharmacology 4: $416-422$.

Morgan D, Liu Y, Roberts DC (2006). Rapid and persistent sensitization to the reinforcing effects of cocaine. Neuropsychopharmacology 31: 121-128.

Nelson A, Killcross S (2006). Amphetamine exposure enhances habit formation. J Neurosci 26: 3805-3812.

Phillips AG, Di Ciano P (1996). Behavioral sensitization is induced by intravenous self-administration of cocaine by rats. Psychopharmacology 124: 279-281.

Robbins TW (1976). Relationship between reward-enhancing and stereotypical effects of psychomotor stimulant drugs. Nature 264: 57-59.

Robbins TW, Everitt BJ (1999). Drug addiction: bad habits add up. Nature 398: $567-570$.

Robinson TE, Berridge KC (1993). The neural basis of drug craving: an incentive-sensitization theory of addiction. Brain Res Brain Res Rev 18: 247-291.

Robinson TE, Berridge KC (2000). The psychology and neurobiology of addiction: an incentive-sensitization view. Addiction 95(Suppl 2): S91-S117.

Schoenbaum G, Saddoris MP, Ramus SJ, Shaham Y, Setlow B (2004). Cocaine-experienced rats exhibit learning deficits in a task sensitive to orbitofrontal cortex lesions. Eur J Neurosci 19: 1997-2002.

Shahan TA (2002). The observing-response procedure: a novel method to study drug-associated conditioned reinforcement. Exp Clin Psychopharmacol 10: 3-9.

Shahan TA, Burke KA (2004). Ethanol-maintained responding of rats is more resistant to change in a context with added non-drug reinforcement. Behav Pharmacol 15: 279-285.

Taylor JR, Horger BA (1999). Enhanced responding for conditioned reward produced by intra-accumbens amphetamine is potentiated after cocaine sensitization. Psychopharmacology (Berl) 142: 31-40.

Tiffany ST (1990). A cognitive model of drug urges and drug-usebehavior: role of automatic and nonautomatic processes. Psychol Rev 97: 147-168.

Vanderschuren LJ, Beemster P, Schoffelmeer AN (2003). On the role of noradrenaline in psychostimulant-induced psychomotor activity and sensitization. Psychopharmacology (Berl) 169: 176-185.

Vanderschuren LJ, Kalivas PW (2000). Alterations in dopaminergic and glutamatergic transmission in the induction and expression of behavioral sensitization: a critical review of preclinical studies. Psychopharmacology (Berl) 151: 99-120.

Vezina P, Lorrain DS, Arnold GM, Austin JD, Suto N (2002). Sensitization of midbrain dopamine neuron reactivity promotes the pursuit of amphetamine. J Neurosci 22: 4654-4662.

Wyvell CL, Berridge KC (2000). Intra-accumbens amphetamine increases the conditioned incentive salience of sucrose reward: enhancement of reward 'wanting' without enhanced 'liking' or response reinforcement. J Neurosci 20: 8122-8130.

Wyvell CL, Berridge KC (2001). Incentive sensitization by previous amphetamine exposure: increased cue- triggered 'wanting' for sucrose reward. J Neurosci 21: 7831-7840.

Zimmerman DW (1957). Durable secondary reinforcement: method and theory. Psychol Rev 64(Part 1): 373-383. 